\title{
Association of left atrial strain by cardiovascular magnetic resonance with recurrence of atrial fibrillation following catheter ablation
}

Mina M. Benjamin ${ }^{1}$, Naeem Moulki ${ }^{1}$, Aneeq Waqar ${ }^{2}$, Harish Ravipati ${ }^{3}$, Nancy Schoenecker ${ }^{1}$, David Wilber ${ }^{1}$, Menhel Kinno ${ }^{1}$, Mark Rabbat ${ }^{1}$, Thriveni Sanagala ${ }^{1}$ and Mushabbar A. Syed ${ }^{1 *}$ (D)

\begin{abstract}
Background: Atrial fibrillation (AF) is a progressive condition, which is characterized by inflammation/fibrosis of left atrial (LA) wall, an increase in the LA size/volumes, and decrease in LA function. We sought to investigate the relationship of anatomical and functional parameters obtained by cardiovascular magnetic resonance (CMR), with AF recurrence in paroxysmal AF (pAF) patients after catheter ablation.

Methods: We studied 80 consecutive pAF patients referred for ablation, between January 2014 and December 2019, who underwent pre- and post-ablation CMR while in sinus rhythm. LA volumes were measured using the area-length method and included maximum, minimum, and pre-atrial-contraction volumes. CMR-derived LA reservoir strain (ER), conduit strain $(\mathcal{E C D})$, and contractile strain $(\mathcal{E C T})$ were measured by computer assisted manual planimetry. We used a multivariate logistical regression to estimate the independent predictors of AF recurrence after ablation.

Results: Mean age was $58.6 \pm 9.4$ years, $75 \%$ men, mean $\mathrm{CHA}_{2} \mathrm{DS}_{2}$-VASc score was 1.7, 36\% had prior cardioversion and $51 \%$ were taking antiarrhythmic drugs. Patients were followed for a median of 4 years (Q1-Q3 $=2.5-6.2$ years). Of the 80 patients, 21 (26.3\%) patients had AF recurrence after ablation. There were no significant differences between AF recurrence vs. no recurrence groups in age, gender, $\mathrm{CHA}_{2} \mathrm{DS}_{2}$-VASc score, or baseline comorbidities. At baseline, patients with AF recurrence compared to without recurrence had lower LV end systolic volume index (32 $\pm 7 \mathrm{vs}$ $37 \pm 11 \mathrm{~mL} / \mathrm{m} 2 ; p=0.045)$ and lower $E C T(7.1 \pm 4.6$ vs $9.1 \pm 3.7 ; p=0.05)$. Post-ablation, patients with AF recurrence had higher LA minimum volume ( $68 \pm 32$ vs $55 \pm 23 ; p=0.05$ ), right atrial volume index ( $62 \pm 20$ vs $52 \pm 19 \mathrm{~mL}$ / $\mathrm{m} 2 ; \mathrm{p}=0.04$ ) and lower $\mathrm{LA}$ active ejection fraction ( $24 \pm 8 \mathrm{vs} 29 \pm 11 ; p=0.05)$, LA total ejection fraction (39 $\pm 14 \mathrm{vs}$ $46 \pm 12 ; p=0.02)$, LA expansion index (73.6 \pm 37.5 vs $94.7 \pm 37.1 ; p=0.03)$ and $E C T(6.2 \pm 2.9$ vs $7.3 \pm 1.7 ; p=0.04)$. Adjusting for clinical variables in the multivariate logistic regression model, post-ablation minimum LA volume (OR 1.09; $\mathrm{Cl} 1.02-1.16)$, LA expansion index (OR 0.98; $\mathrm{Cl} 0.96-0.99)$, and baseline $\mathcal{E} R(\mathrm{OR} 0.92 ; \mathrm{Cl} 0.85-0.99$ ) were independently associated with AF recurrence.
\end{abstract}

Conclusion: Significant changes in LA volumes and strain parameters occur after AF ablation. CMR derived baseline $\mathcal{E R}$, post-ablation minimum $L A V$, and expansion index are independently associated with $A F$ recurrence.

\footnotetext{
*Correspondence: masyed@lumc.edu

${ }^{1}$ Division of Cardiovascular Medicine, Loyola University Medical Center, Stritch School of Medicine, Maywood, IL, USA

Full list of author information is available at the end of the article
}

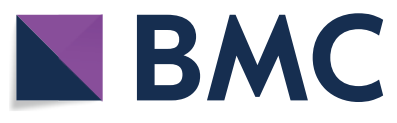

(c) The Author(s) 2021. Open Access This article is licensed under a Creative Commons Attribution 4.0 International License, which permits use, sharing, adaptation, distribution and reproduction in any medium or format, as long as you give appropriate credit to the original author(s) and the source, provide a link to the Creative Commons licence, and indicate if changes were made. The images or other third party material in this article are included in the article's Creative Commons licence, unless indicated otherwise in a credit line to the material. If material is not included in the article's Creative Commons licence and your intended use is not permitted by statutory regulation or exceeds the permitted use, you will need to obtain permission directly from the copyright holder. To view a copy of this licence, visit http://creativecommons.org/licenses/by/4.0/. The Creative Commons Public Domain Dedication waiver (http://creativeco mmons.org/publicdomain/zero/1.0/) applies to the data made available in this article, unless otherwise stated in a credit line to the data. 
Keywords: Atrial fibrillation, Left atrial strain, Cardiovascular magnetic resonance, Catheter ablation, Recurrence

\section{Background}

Atrial fibrillation (AF) is the most common arrhythmia and can have adverse consequences related to reduction in cardiac output and atrial appendage thrombus formation. Affected patients may also be at increased risk for mortality $[1,2]$. AF is also a progressive condition that is associated with structural and electrical changes in the left atrium (LA). LA remodelling in AF patients is characterized by LA wall inflammation and fibrosis, an increase in the LA size/volumes, and decrease in LA function [3]. Dynamic changes in the LA volumes during ventricular systole, diastole and atrial contraction are represented as reservoir, conduit and booster/contractile function respectively. These LA phasic volumes are influenced by cardiac rhythm and most accurate measurements of LA volumes are performed when patients are in sinus rhythm [4]. These LA parameters can be measured by two dimensional echocardiography (2DE), three dimensional echocardiography (3DE), tissue Doppler imaging, cardiac computed tomography and cardiovascular magnetic resonance (CMR) of which CMR is considered the gold standard [5-8].

For patients with AF in whom a rhythm control strategy is chosen, catheter ablation through pulmonary vein isolation (PVI) is a principal therapeutic intervention to reduce the frequency or eliminate episodes of AF. Some studies have reported that PVI leads to a positive change in LA anatomical and functional parameters which may correlate with AF recurrence [9-11]. CMR strain by tissue tracking is a novel, relatively simple and reproducible technique that is being investigated for assessment of atrial mechanics [5, 12-15]. Standardized strain parameters include LA reservoir strain (ER), contractile strain $(E C T)$, and conduit strain (ECD) [16]. We sought to investigate the association of CMR-derived LA anatomical and functional parameters including strain with $\mathrm{AF}$ recurrence post catheter ablation in patients with paroxysmal AF (pAF). We hypothesized that changes in LA function and/or strain as assessed by CMR are associated with AF recurrence after catheter AF ablation.

\section{Methods}

\section{Patient population}

This study was approved by Loyola University Medical Centre's Institutional Review Board. The inclusion criteria were: (1) patients diagnosed with pAF, (2) underwent a catheter-based PVI between January 2014 and December 2019, (3) underwent pre- and post-ablation CMR.
Patients were excluded if they had: (1) implanted pacemaker or implanted cardiovert-defibrillator device, (2) non-sinus rhythm during CMR, (3) were lost to follow up within a year of the ablation procedure, or (4) history of heart transplant. A total of 102 patients met our inclusion criteria. After excluding patients where CMR strain was uninterpretable $(n=15)$ or those who were lost to follow up $(n=7), 80$ patients were included in this analysis. In addition to PVI, patients underwent ablation of LA rotors $(n=13)$, cavotricuspid isthmus $(n=22)$, superior vena cava $(n=5)$ and roof line ablation $(n=8)$.

\section{CMR imaging and analysis}

CMR was performed prior to (median 32 days; Q1Q3: 15-67 days) and after (median 131 days; Q1-Q3: 114-201 days) AF ablation. CMR images were acquired using clinically available retrospective electrocardiogram (ECG) gated balanced steady-state free precession sequence on $3 \mathrm{~T}$ CMR scanner (Magnetom Trio, Siemens Healthineers, Erlangen, Germany). CMR acquisition parameters included a temporal resolution of $\approx 50 \mathrm{~ms}$, TR $45 \mathrm{~ms}$, TE $1.2 \mathrm{~ms}$, FOV $300-360 \mathrm{~cm}$, matrix $224 \times 204$, and slice thickness $7 \mathrm{~mm}$. CMR quantitative analysis was performed on cvi42 (version 5.0; Circle Cardiovascular Imaging Inc., Calgary, Alberta, Canada) using computer assisted manual planimetry. LA volumes were measured using the arealength method (4) and included maximum, minimum, and pre-atrial-contraction volumes from the 2 and 4 chamber views, volume $=(0.848 \times$ area $4 \mathrm{ch} \times$ area2ch $) /$ ([length2ch + length4ch]/2). All measured LA volumes were indexed to body surface area. The variables of LA volumes (LAV) included in the study were:

- Maximum LA Volume (LAVmax)=LA volume at end systole before mitral valve opening

- Minimum LA Volume (LAVmin)=LA volume at end diastole right after mitral valve closure

- Preatrial Contraction LA volume (LAVPreA) $=$ LA volume before atrial contraction

Using the measured LA volumes at different points of cardiac cycle, LA ejection fraction (LAEF) was calculated as follows:

- Passive LAEF: (LAVmax-LAVPreA)/LAVmax.

- Active LAEF: (LAVPreA-LAVmin)/LAVPreA.

- Total LAEF: (LAVmax-LAVmin)/LAVmax.

- Expansion index: (LAVmax-LAVmin)/LAVmin 
Multimodality Tissue Tracking software (version 5.0, Circle Cardiovascular Imaging Inc.) was used to measure $\varepsilon R$ and ECT from 4- and 2-chamber cine CMR images (Fig. 1), the difference between these two measurements was ECD [16]. Strain measurements were performed by computer assisted manual planimetry where the investigator defines endocardial and epicardial borders of the LA at end systole and using the marked points, the software creates endocardial and epicardial borders and then tracks LA wall in subsequent frames. CINE loops with superimposed longitudinal strain tracings were played to confirm accurate tracking of the LA endo- and epicardium throughout the cardiac cycle. If tracking was inaccurate at some point during the cardiac cycle, the planimetry was corrected manually and the software algorithm reapplied. The final strain results were taken as an average of the 2 and 4 chamber views. This method has been previously validated with excellent reproducibility (intraclass correlation coefficients between 0.90 and 0.97 for LA volumes and strain) [16-18]. Strain measurements were accepted only in the case of adequate tracking quality in at least five of the six segments per view. We also calculated $\Delta E R, \Delta E C T, \Delta E C D$ as the difference between pre- and post-ablation values of $\varepsilon R, \varepsilon C T$ and $\varepsilon C D$, respectively. Strain measurements were performed by a CMR trained and board-certified investigator with one year experience who was blinded to the case status of the participants. All patients were followed up in outpatient clinic for monitoring and arrhythmia management. AF recurrence was identified by ECG documentation of an atrial tachyarrhythmia lasting $\geq 30 \mathrm{~s}$ on a 12-lead ECG, event recording, or Holter monitor recording. Recurrence rates were determined regardless of whether patients were on antiarrhythmic medications or not. Atrial arrhythmias that occurred during the first 90 days after catheter ablation and resolved spontaneously or with cardioversion, were not counted as recurrences (blanking period).

\section{Statistical analysis}

Continuous variables are presented as mean $\pm S D$ if normally distributed and as medians with interquartile ranges (Q1-Q3) if not normally distributed. Categorical variables are presented as frequencies and percentages. Differences between group means were evaluated with $t$ tests for continuous variables or $\chi^{2}$ analysis for

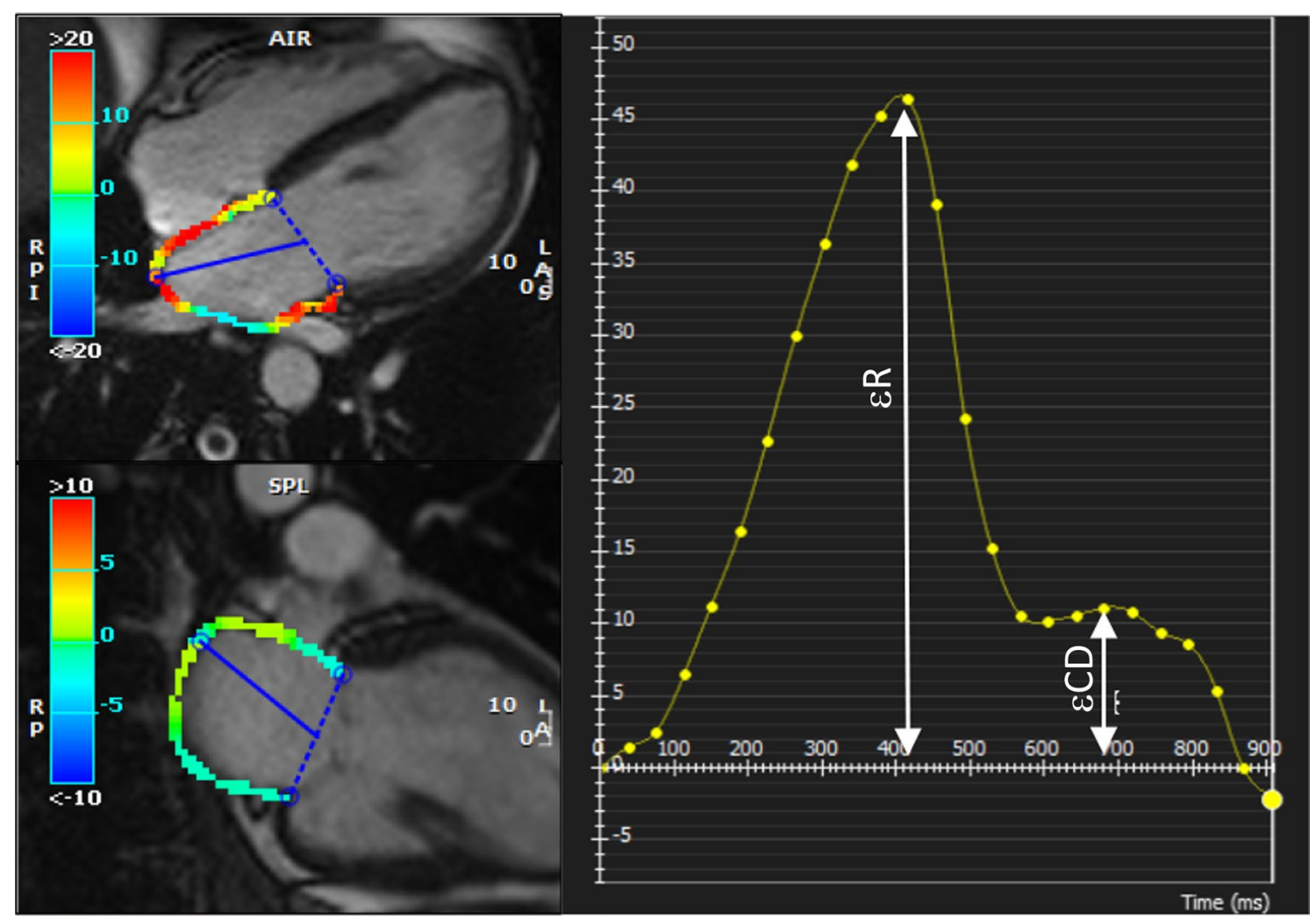

Fig. 1 Left: Example of left atrial (LA) tissue tracking in still frames of cine cardiovascular magnetic resonance (CMR) (balanced steady-state free precession imaging) four chamber (left top) and two chamber (left bottom) cine loops. Right: Longitudinal strain with two peaks representing reservoir $(\boldsymbol{E R})$ and contractile $(\boldsymbol{E} C T)$ strain. The difference between the two measurements is conduit strain $(\boldsymbol{E} C D)$ 
categorical variables. Multivariate logistic regression model was constructed adjusting for the following clinical parameters: age, gender, hypertension, obstructive sleep apnea in addition to the CMR-derived LA parameters with $\mathrm{P}$ values $<0.1$. To avoid collinearity, correlations between continuous variables were tested using the Spearman correlation coefficient and variables with $\mathrm{r}>0.50$ were not included in the model. We used a modified Cox proportional hazard model to estimate the timedependent hazard ratios of AF recurrence in tertiles of patients based on baseline ER and post-ablation ECT. Cox models were adjusted for the same predictors of AF recurrence as used for the multivariable model, mentioned above. Statistical analyses were performed using MedCalc for Windows (version 15.0, MedCalc Software, Ostend, Belgium). All P values reported are from twosided tests, and $\mathrm{P}$ values $<0.05$ were considered statistically significant.

\section{Results}

Baseline demographics, clinical characteristics and medications at the time of the ablation procedure are listed in Table 1. Mean age was $58.6 \pm 9.4$ years, $75 \%$ were men, mean $\mathrm{CHA}_{2} \mathrm{DS}_{2}$-VASc score was $1.7 \pm 1.4 ; 36 \%$ had prior cardioversion and $51 \%$ were taking antiarrhythmic drugs. Patients were followed for a median of 4 years (Q1$\mathrm{Q} 3=2.5-6.2$ years) after AF ablation. Of 80 patients, 21 (26.3\%) patients had AF recurrence. The median duration after catheter ablation to AF recurrence was 5 months (Q1-3: 3-11 months). There were no significant differences between $\mathrm{AF}$ recurrence vs. no $\mathrm{AF}$ recurrence groups in age, gender, $\mathrm{CHA}_{2} \mathrm{DS}_{2}$-VASc score, or baseline comorbidities (all $\mathrm{P}>0.05$ ).

$\mathrm{AF}$ ablation was associated with a decrease in LA volumes (LAVmax, LAVmin, LAVpreA), pulmonary vein ostial diameters and all three LA strain parameters (Table 2). No significant improvement was seen in right atrial (RA) volumes or left ventricular (LV) and right ventricular (RV) volumes and ejection fraction.

At baseline, patients with AF recurrence (Table 3) had lower LV end systolic volume index $(\mathrm{p}=0.045)$ and lower ECT $(\mathrm{p}=0.05)$. Post-ablation, patients with AF recurrence had higher LA minimum volume $(p=0.05)$, RA volume index $(p=0.04)$ and lower LA active ejection fraction $(\mathrm{p}=0.05)$, LA total ejection fraction $(\mathrm{p}=0.02)$, LA expansion index $(\mathrm{p}=0.03)$ and ECT $(\mathrm{p}=0.04)$.

Adjusting for clinical variables in the multivariate logistic regression model, post-ablation minimum LAV (OR 1.09; CI 1.02-1.16), LA expansion index (OR 0.98; CI 0.96-0.99), and baseline ER (OR 0.92; CI 0.85-0.99) were independently associated with AF recurrence. (Table 4) Cox proportional hazard model results are presented in Figs. 2 and 3 where survival free of AF is represented for the three tertiles of the baseline $\mathcal{E}$ and post-ablation $\varepsilon C T$. For the baseline $E R$ model, tertiles one, two, and three corresponded to a baseline $E R$ of $<21.5,21.5-27$ and $>27$, respectively. The model showed a longer survival free of AF in patients with a higher baseline ER. For the post-ablation $E C T$ model, teriles one, two, and three corresponded to a post-ablation ECT of $<5.3,5-8$ and $>8$, respectively. The model showed a trend towards longer survival free of AF in patients with higher post-ablation ECT. Additional file 1 shows comparison graphs of baseline and post-ablation reservoir and contractile strain in $\mathrm{AF}$ recurrence and no-recurrence groups.

\section{Discussion}

In this retrospective study, we evaluated the association of CMR derived LA anatomical and functional parameters in pAF patients with AF recurrence after catheter ablation. Our main finding were that: (1) PVI leads to a decrease in LA volumes with a decrease in all LA strain parameters (2) baseline $E R$ and post-ablation minimum LAV and LA expansion index are independently associated with AF recurrence.

AF recurrence is not uncommon after catheter ablation with freedom from atrial arrhythmia at long-term followup ( $\geq 2$ years) after a single procedure is about $53 \%$ which increases to $80 \%$ with multiple procedures [19]. Early recurrence is thought to be due to leaking of AF electrical impulses due to less early fibrous tissue formation around pulmonary veins after PVI whereas late recurrence is more likely from the shifting of AF foci from pulmonary veins to the LA due to stressors and other comorbid conditions [20]. Poor LA function has been associated with several poor outcomes including implantable cardioverter defibrillator (ICD) shocks [17, 18, 21-23], ischemic events and worsening LV diastolic function in patients [22]. LA size and volumes have been reported as predictors of sinus rhythm maintenance after AF ablation [24, 25]. LA size using echocardiographic derived anteroposterior LA diameter has been studied mostly. A meta-analysis by Zhuang et al. of 22 studies (3750 subjects) that used anteroposterior LA diameter showed that the mean difference of LA diameter between patients with and without recurrence was $1.9 \mathrm{~mm}$ (95\% CI 1.3$2.5, \mathrm{P}<0.001)$ and dilated LA was associated with significantly increased risk of AF recurrence after single PVI [24]. LAV is more accurate in assessing LA size but studies using LAV as a predictor of AF recurrence have been contradictory. Njoku et al. published a meta-analysis of 21 studies (3822 subjects) that used LAV/LAV index as a predictor of AF recurrence after PVI [25]. Both mean LAV (11 studies, 1559 subjects) and LAV index (9 studies, 1425 subjects) were higher in patients with recurrence (OR 1.03, CI 1.01-1.05). Our study had a more 
Table 1 Patient baseline characteristics

\begin{tabular}{|c|c|c|c|c|}
\hline & Overall $n=80$ & No AF recurrence $n=59$ & AF recurrence $n=21$ & $p$-value \\
\hline \multicolumn{5}{|l|}{ Demographics } \\
\hline Age, mean $\pm S D$ & $58.6 \pm 9.4$ & $58.5 \pm 11.5$ & $58.6 \pm 8.9$ & 0.99 \\
\hline Male & $60(75)$ & $42(71.2)$ & $18(85.7)$ & 0.2 \\
\hline Caucasian & $76(95)$ & $54(91.5)$ & $21(100)$ & 0.64 \\
\hline Prior DC cardioversion & $29(36.3)$ & $23(39)$ & $6(28.6)$ & 0.2 \\
\hline \multicolumn{5}{|l|}{ Comorbidities } \\
\hline Current/former smoker & $20(25)$ & $16(27.1)$ & $4(19)$ & 0.29 \\
\hline Coronary artery disease & $10(12.5)$ & $9(15.3)$ & $1(4.8)$ & 0.16 \\
\hline Peripheral arterial disease & $1(1.25)$ & 0 & $1(4.8)$ & 0.12 \\
\hline Heart failure & $8(10)$ & $7(11.9)$ & $1(4.8)$ & 0.28 \\
\hline Hypertension & $38(47.5)$ & $27(45.8)$ & $11(52.4)$ & 0.92 \\
\hline Obstructive sleep apnea & $18(22.5)$ & $11(18.6)$ & $7(33.3)$ & 0.33 \\
\hline Diabetes mellitus & $3(3.8)$ & $3(5.1)$ & 0 & 0.27 \\
\hline Cerebrovascular disease & $1(1.25)$ & $1(1.7)$ & 0 & 0.53 \\
\hline \multicolumn{5}{|l|}{$\mathrm{CHA}_{2} \mathrm{DS}_{2}$-VASc score } \\
\hline 0 & $22(27.5)$ & $16(27.1)$ & $6(28.6)$ & 0.79 \\
\hline 1 & $32(40)$ & $21(35.6)$ & $11(52.4)$ & 0.45 \\
\hline 2 & $16(20)$ & $13(22)$ & $3(14.3)$ & 0.30 \\
\hline$\geq 3$ & $10(12.5)$ & $7(11.9)$ & $3(14.3)$ & 0.61 \\
\hline \multicolumn{5}{|l|}{ Medications } \\
\hline Aspirin & $17(21.3)$ & $11(18.6)$ & $6(28.6)$ & 0.57 \\
\hline Statin & $29(36.3)$ & $1(1.7)$ & $2(9.5)$ & 0.15 \\
\hline Warfarin & $9(11.3)$ & $7(11.9)$ & $2(9.5)$ & 0.62 \\
\hline Apixaban & $12(15)$ & $9(15.3)$ & $3(14.3)$ & 0.73 \\
\hline Rivaroxaban & $35(43.8)$ & $23(39.0)$ & $12(57.1)$ & 0.43 \\
\hline Dabigatran & $4(5)$ & $3(5.1)$ & $1(4.8)$ & 0.85 \\
\hline Beta Blocker & $55(68.8)$ & $40(67.8)$ & $15(71.4)$ & 0.52 \\
\hline Calcium channel blocker & $22(27.5)$ & $18(30.5)$ & $4(19)$ & 0.18 \\
\hline Anti-Arrhythmic Therapy & $45(56.3)$ & $32(54.2)$ & $13(61.9)$ & 0.33 \\
\hline Flecanaide & $17(21.3)$ & $10(16.9)$ & $7(33.3)$ & 0.25 \\
\hline Propafenone & $4(5)$ & $3(5.1)$ & $1(4.8)$ & 0.85 \\
\hline Sotalol & $4(5)$ & $1(1.7)$ & $3(14.3)$ & 0.04 \\
\hline Amiodarone & $4(5)$ & $4(6.8)$ & 0 & 0.20 \\
\hline Dronderone & $14(17.5)$ & $11(18.6)$ & $3(14.3)$ & 0.48 \\
\hline ACE/ARB & $22(27.5)$ & $19(32.2)$ & $3(14.3)$ & 0.06 \\
\hline Levothyroxine & $7(8.8)$ & $4(6.8)$ & $3(14.3)$ & 0.42 \\
\hline Diuretic & $14(17.5)$ & 11 (18.6) & $3(14.3)$ & 0.48 \\
\hline
\end{tabular}

Significant p-values are shown in bold

$A C E$, angiotensin converting enzyme inhibitor; $A R B$, angiotensin receptor blocker; $D C$, direct current

comprehensive design and evaluated both volumes and LA function parameters pre and post ablation as predictors of AF recurrence after PVI.

LAV measured in our study were higher than normal values by CMR reported in the literature [26]. Considering that our study only included patients with pAF, this is not an unexpected finding. Our LAV were fairly similar to previous studies in patients with $\operatorname{AF}[9,10]$. LA remodelling may occur after PVI and entails a decrease in
LAV and an increase in LAEF [10]. This LA remodelling has been associated with AF recurrence, more so with late than early recurrence [27]. Recent data from MultiEthnic Study of Atherosclerosis (MESA) study showed that adverse remodelling i.e. elevated LAV and decreased passive and total LAEF, precedes AF in a multi-ethnic population who were free of clinical cardiovascular disease at baseline [26]. 
Table 2 Comparison of pre and post ablation LA characteristics on CMR

\begin{tabular}{|c|c|c|c|}
\hline & Pre-ablation & Post-ablation & $p$-value \\
\hline LAV maximum $(\mathrm{mL})$ & $112 \pm 36$ & $96 \pm 30$ & 0.003 \\
\hline LAV maximum index $\left(\mathrm{mL} / \mathrm{m}^{2}\right)$ & $52 \pm 18$ & $45 \pm 14$ & 0.01 \\
\hline LAV minimum (mL) & $63 \pm 32$ & $53 \pm 26$ & 0.03 \\
\hline LAV minimum index $\left(\mathrm{mL} / \mathrm{m}^{2}\right)$ & $29 \pm 16$ & $25 \pm 12$ & 0.046 \\
\hline LAV pre-A $(\mathrm{mL})$ & $86 \pm 35$ & $73 \pm 27$ & 0.007 \\
\hline LAV pre-A index (mL/m²) & $40 \pm 17$ & $34 \pm 13$ & 0.02 \\
\hline LA passive EF (\%) & $25 \pm 12$ & $24 \pm 9$ & 0.59 \\
\hline LA active EF (\%) & $29 \pm 15$ & $27 \pm 16$ & 0.31 \\
\hline LA total EF (\%) & $46 \pm 15$ & $43 \pm 15$ & 0.25 \\
\hline LA expansion index & $95 \pm 51$ & $88 \pm 49$ & 0.41 \\
\hline LVEF & $60 \pm 9$ & $57 \pm 6$ & 0.1 \\
\hline LVEDVI $\left(\mathrm{mL} / \mathrm{m}^{2}\right)$ & $86 \pm 16$ & $90 \pm 25$ & 0.33 \\
\hline LVESVI (mL/m²) & $36 \pm 11$ & $39 \pm 11$ & 0.1 \\
\hline LV mass (mg) & $97 \pm 33$ & $88 \pm 27$ & 0.07 \\
\hline RVEF & $53 \pm 6$ & $51 \pm 6$ & 0.09 \\
\hline RVEDVI $\left(\mathrm{mL} / \mathrm{m}^{2}\right)$ & $94 \pm 22$ & $93 \pm 22$ & 0.89 \\
\hline $\operatorname{RVESVI}\left(\mathrm{mL} / \mathrm{m}^{2}\right)$ & $45 \pm 14$ & $46 \pm 13$ & 0.48 \\
\hline RA volume (mL) & $143 \pm 40$ & $132 \pm 39$ & 0.07 \\
\hline RA volume index & $68 \pm 19$ & $65 \pm 19$ & 0.32 \\
\hline RUPV diameter (mm) & $19.5 \pm 3.2$ & $16.9 \pm 3.8$ & $<0.001$ \\
\hline RLPV diameter (mm) & $17.9 \pm 3.1$ & $16.6 \pm 3.1$ & 0.01 \\
\hline LUPV diameter (mm) & $15.2 \pm 2.5$ & $12.9 \pm 3.2$ & $<0.001$ \\
\hline LLPV diameter (mm) & $13.9 \pm 2.1$ & $11.7 \pm 4.5$ & 0.03 \\
\hline$\varepsilon R$ & $24.0 \pm 10.4$ & $19.8 \pm 8.1$ & 0.005 \\
\hline$\varepsilon \subset T$ & $8.4 \pm 0.1$ & $5.7 \pm 5.3$ & 0.001 \\
\hline$\varepsilon C D$ & $15.6 \pm 7.1$ & $13.0 \pm 6.3$ & 0.02 \\
\hline
\end{tabular}

Significant $\mathrm{p}$-values are shown in bold

$E D V$, end diastolic volume; EF, ejection fraction; ESV, end systolic volume; LA left atrium; LAV, left atrial volume; LLPV, left lower pulmonary vein; LUPV, left upper pulmonary vein; LV, left ventricle; LVEDVI, left ventricular end-diastolic volume index; LVESVI, left ventricular end-systolic volume index; RA, right atrium; RLPV, right lower pulmonary vein; RUPV, right upper pulmonary vein; $\mathrm{RV}$, right ventricle; RVEDVI, right ventricular end-diastolic volume index; RVESVI, right ventricular end-systolic volume index; $\mathcal{E} C D$, conduit strain; $E C T$, contractile strain; ER, reservoir strain

LA strain is a highly reproducible [16] measure of LA deformation and has been shown to be a sensitive marker of intracavitary pressures and LA reservoir function [28]. LA strain is also a useful marker of AF risk incremental to LA size in the general population and is an independent predictor of AF recurrence $[28,29]$.

In our study, baseline ER was significantly associated with AF recurrence in the multivariate analysis. This is in line with previous echocardiographic studies that showed an association between the baseline strain and AF recurrence following PVI or cardioversion [12, 30]. We also found a significant association of post ablation LAVmin and expansion index with AF recurrence after PVI, however, the association was stronger with baseline $E R$ than volumetric indices. Baseline $E R$ would also be more relevant clinically to help risk stratify patients prior to ablation. Studies using echocardiography have shown that functional parameters of LA strain and strain rate on tissue Doppler imaging or speckle echocardiography are better predictors of AF recurrence than LAV [30-33]. Theoretically, LA strain is less affected by loading conditions and by tethering effects than volumetric measurements [22]. Our study extends these findings to CMR as a potential tool for estimating future risk of AF recurrence by using $E R$ prior to ablation.

\section{Limitations}

Several limitations need to be considered when interpreting the results of our study. Our study is a ret $r$ osp ective observational study, with some inherent biases related to the study design including a lack of standardization in patient follow up clinic visits and rhythm monitoring strategies to detect AF recurrence. As described in the methods section, some patients underwent additional ablation in addition to the standard PVI and that might have affe cted the post-ablation LA anatomic and functional parameters. We only included pAF patients who have undergone radiofrequency ablation. Our results $\mathrm{m}$ i ght $\mathrm{n}$ ot be generalizable to those with persistent AF or those who underwent other forms of ablation, e.g. cryoablation. Due to the relatively small sample size, some of the variables may not have reached statistical significance. Although post-ablation CMR is a common routine practice at our institution, selection bias might be significant as we only included patients who were in sinus rhythm at the time of pre and post-ablation CMR. We used clinically available CMR cine sequence with a temporal resolution of $50 \mathrm{~ms}$ for strain measurements based on previously published data by MESA investigators [21]. A higher temporal resolution might have produced more precise measurements. There is no dedicated validated CMR software exclusively for LA strain measurements. We utilized the strain software developed for LV strain as done by other investigators. LA late gadolinium enhancement noted on CMR is an important parameter of LA fibrosis that might have a prognostic factor for AF recurrence, however, it was not investigated in this study.

\section{Conclusions}

We investigated structural and functional parameters of LA in patients with pAF undergoing PVI and found that baseline ER and post-ablation minimum LAV and expansion index were independently associated with $\mathrm{AF}$ recurrence. Among these parameters, baseline ER would be more clinically useful as most patients don't routinely undergo post ablation CMR. Patients with a higher 
Table 3 Pre- and post-ablation CMR characteristics of patients with no AF recurrence versus those with AF recurrence

\begin{tabular}{|c|c|c|c|c|}
\hline & Total $\mathbf{N}=\mathbf{8 0}$ & No $A F$ recurrence $n=59$ & AF recurrence $n=21$ & p-value \\
\hline \multicolumn{5}{|l|}{ Pre-ablation } \\
\hline CMR before ablation procedure (median days, Q1-Q3) & $32(15-67)$ & $26(12-64)$ & $38(99-306)$ & 0.45 \\
\hline LAV maximum $(\mathrm{mL})$ & $112 \pm 36$ & $110 \pm 34$ & $117 \pm 43$ & 0.45 \\
\hline LAV maximum index $\left(\mathrm{mL} / \mathrm{m}^{2}\right)$ & $52 \pm 18$ & $46 \pm 16$ & $47 \pm 16$ & 0.51 \\
\hline LAV minimum $(\mathrm{mL})$ & $63 \pm 32$ & $60 \pm 30$ & $70 \pm 38$ & 0.21 \\
\hline LAV minimum index $\left(\mathrm{mL} / \mathrm{m}^{2}\right)$ & $29 \pm 16$ & $28 \pm 16$ & $33 \pm 16$ & 0.24 \\
\hline LAV pre-A $(\mathrm{mL})$ & $86 \pm 35$ & $82 \pm 33$ & $93 \pm 41$ & 0.21 \\
\hline LAV pre-A index $\left(\mathrm{mL} / \mathrm{m}^{2}\right)$ & $40 \pm 17$ & $38 \pm 18$ & $43 \pm 17$ & 0.24 \\
\hline LA passive ejection fraction (\%) & $25 \pm 12$ & $23 \pm 9$ & $24 \pm 8$ & 0.71 \\
\hline LA active ejection fraction (\%) & $29 \pm 15$ & $30 \pm 16$ & $26 \pm 14$ & 0.30 \\
\hline LA total ejection fraction (\%) & $46 \pm 15$ & $45 \pm 11$ & $43 \pm 13$ & 0.47 \\
\hline LA expansion index & $95 \pm 51$ & $168 \pm 524$ & $87 \pm 58$ & 0.46 \\
\hline LV ejection fraction (\%) & $60 \pm 9$ & $60 \pm 10$ & $61 \pm 4$ & 0.93 \\
\hline LVEDVi $\left(\mathrm{mL} / \mathrm{m}^{2}\right)$ & $86 \pm 16$ & $82 \pm 17$ & $88 \pm 15$ & 0.11 \\
\hline $\operatorname{LVESVi}\left(\mathrm{mL} / \mathrm{m}^{2}\right)$ & $36 \pm 11$ & $32 \pm 7$ & $37 \pm 11$ & 0.05 \\
\hline LV mass (mg) & $97.0 \pm 33.4$ & $95.1 \pm 32.3$ & $102.4 \pm 36.7$ & 0.38 \\
\hline RVEF (\%) & $53 \pm 6$ & $52 \pm 6$ & $55 \pm 7$ & 0.12 \\
\hline RVEDVi $\left(\mathrm{mL} / \mathrm{m}^{2}\right)$ & $94 \pm 22$ & $95 \pm 22$ & $91 \pm 24$ & 0.55 \\
\hline RVESVi $\left(m L / m^{2}\right)$ & $45 \pm 14$ & $44 \pm 10$ & $45 \pm 22$ & 0.89 \\
\hline RA volume (mL) & $143 \pm 40$ & $139 \pm 38$ & $155 \pm 48$ & 0.12 \\
\hline $\operatorname{RAVI}\left(\mathrm{mL} / \mathrm{m}^{2}\right)$ & $68 \pm 19$ & $67 \pm 19$ & $70 \pm 20$ & 0.55 \\
\hline$\varepsilon R$ & $24.0 \pm 10.4$ & $25.2 \pm 10.7$ & $20.8 \pm 8.9$ & 0.06 \\
\hline$\varepsilon \subset T$ & $8.4 \pm 4.1$ & $9.1 \pm 3.7$ & $7.1 \pm 4.6$ & 0.05 \\
\hline$\varepsilon C D$ & $15.6 \pm 7.1$ & $16.2 \pm 7.7$ & $13.7 \pm 4.9$ & 0.17 \\
\hline \multicolumn{5}{|l|}{ Post-ablation } \\
\hline CMR after ablation procedure (median days, Q1-Q3) & $131(114-201)$ & $140(99-306)$ & $124(96-151.5)$ & 0.31 \\
\hline LA maximum volume $(\mathrm{mL})$ & $96 \pm 30$ & $100 \pm 27$ & $104 \pm 36$ & 0.57 \\
\hline LAV maximum index $\left(\mathrm{mL} / \mathrm{m}^{2}\right)$ & $45 \pm 14$ & $47 \pm 14$ & $47 \pm 16$ & 0.85 \\
\hline LAV minimum $(\mathrm{mL})$ & $53 \pm 23$ & $55 \pm 22$ & $68 \pm 26$ & 0.05 \\
\hline LAV minimum index $\left(\mathrm{mL} / \mathrm{m}^{2}\right)$ & $25 \pm 12$ & $26 \pm 8$ & $31 \pm 11$ & 0.06 \\
\hline LAV pre-A $(\mathrm{mL})$ & $73 \pm 27$ & $77 \pm 25$ & $79 \pm 32$ & 0.74 \\
\hline LAV pre-A index $\left(\mathrm{mL} / \mathrm{m}^{2}\right)$ & $34 \pm 13$ & $37 \pm 12$ & $36 \pm 14$ & 0.79 \\
\hline LA passive EF (\%) & $24 \pm 9$ & $24 \pm 9$ & $24 \pm 8$ & 0.96 \\
\hline LA active EF (\%) & $27 \pm 16$ & $29 \pm 11$ & $24 \pm 8$ & 0.05 \\
\hline LA total EF (\%) & $43 \pm 15$ & $46 \pm 12$ & $39 \pm 14$ & 0.02 \\
\hline LA expansion index & $88 \pm 49$ & $95 \pm 37$ & $74 \pm 38$ & 0.03 \\
\hline $\operatorname{LVEF}(\%)$ & $57 \pm 6$ & $58 \pm 6$ & $60 \pm 3$ & 0.85 \\
\hline LVEDVI $\left(\mathrm{mL} / \mathrm{m}^{2}\right)$ & $90 \pm 25$ & $85 \pm 19$ & $88 \pm 38$ & 0.79 \\
\hline LVESVI $\left(\mathrm{mL} / \mathrm{m}^{2}\right)$ & $39 \pm 11$ & $36 \pm 12$ & $34 \pm 8$ & 0.77 \\
\hline LV mass (mg) & $88.2 \pm 27.0$ & $94.2 \pm 26.6$ & $96.1 \pm 28.8$ & 0.69 \\
\hline RVEF (\%) & $51 \pm 6$ & $52 \pm 6$ & $53 \pm 5$ & 0.42 \\
\hline RVEDVI $\left(\mathrm{mL} / \mathrm{m}^{2}\right)$ & $93 \pm 22$ & $89 \pm 21$ & $87 \pm 24$ & 0.86 \\
\hline RVESVI $\left(m L / m^{2}\right)$ & $46 \pm 13$ & $43 \pm 13$ & $39 \pm 11$ & 0.81 \\
\hline RA volume (mL) & $132 \pm 39$ & $121 \pm 38$ & $137 \pm 42$ & 0.11 \\
\hline $\operatorname{RAVI}\left(\mathrm{mL} / \mathrm{m}^{2}\right)$ & $65 \pm 19$ & $52 \pm 18$ & $62 \pm 20$ & 0.04 \\
\hline$\varepsilon R$ & $21.4 \pm 8.1$ & $21.4 \pm 7.8$ & $21.0 \pm 9.1$ & 0.77 \\
\hline$\varepsilon \subset T$ & $7.0 \pm 5.3$ & $7.3 \pm 1.7$ & $6.2 \pm 2.9$ & 0.04 \\
\hline$\varepsilon C D$ & $14.4 \pm 6.3$ & $14.1 \pm 6.2$ & $14.8 \pm 7.2$ & 0.3 \\
\hline$\Delta \varepsilon R$ & $2.7 \pm 9.3$ & $3.8 \pm 9.1$ & $-0.2 \pm 9.7$ & 0.09 \\
\hline$\triangle \mathcal{E} C T$ & $1.4 \pm 4.5$ & $1.7 \pm 4.2$ & $0.9 \pm 5.3$ & 0.49 \\
\hline$\triangle E C D$ & $1.3 \pm 6.6$ & $2.1 \pm 6.7$ & $-1.1 \pm 6.2$ & 0.06 \\
\hline
\end{tabular}


Table 3 (continued)

\section{Significant p-values are shown in bold}

$E D V$, end diastolic volume; EF, ejection fraction; ESV, end systolic volume; LA, left atrium; LAV, left atrial volume; LLPV, left lower pulmonary vein; LUPV, left upper pulmonary vein; $L V$, left ventricle; $R A$, right atrium; $R L P V$, right lower pulmonary vein; $R U P V$, right upper pulmonary vein; $R V$, right ventricle; $E C D$, conduit strain; $E C T$, contractile strain; $\mathcal{E}$, reservoir strain, $\triangle E R, \triangle E C T, \triangle E C D$, difference between pre- and post-ablation values of $E R, E C T$ and $E C D$, respectively

Table 4 Univariate (unadjusted) and multi-variate (adjusted) logistic regression results for factors associated with AF recurrence

\begin{tabular}{|c|c|c|c|c|c|c|}
\hline Variable & $\begin{array}{l}\text { Unadjusted } \\
\text { odds ratio }\end{array}$ & Confidence interval & P value & $\begin{array}{l}\text { Adjusted } \\
\text { odds ratio }\end{array}$ & Confidence interval & $P$ value \\
\hline Age & 1.01 & $0.96-1.06$ & 0.57 & 1.03 & $0.95-1.09$ & 0.47 \\
\hline Female gender & 2.4 & $0.6-9.3$ & 0.29 & 1.9 & $0.49-6.7$ & 0.33 \\
\hline Hypertension & 1.15 & $0.43-3.13$ & 0.44 & 1.19 & $0.57-2.58$ & 0.26 \\
\hline Obstructive sleep apnea & 1.96 & $0.61-6.29$ & 0.09 & 3.2 & $0.68-15.01$ & 0.07 \\
\hline LAVmax (post-ablation) & 1.00 & $0.95-1.02$ & 0.19 & 0.94 & $0.89-1.0$ & 0.05 \\
\hline LAVmin (post-ablation) & 1.02 & $1.00-1.04$ & 0.06 & 1.09 & $1.02-1.16$ & 0.01 \\
\hline LA expansion index (post-ablation) & 0.98 & $0.97-0.99$ & 0.04 & 0.98 & $0.96-0.99$ & 0.02 \\
\hline Baseline $\mathcal{E R}$ & 0.94 & $0.86-0.99$ & 0.04 & 0.92 & $0.85-0.99$ & 0.03 \\
\hline Post-ablation ER & 1.02 & $0.95-1.07$ & 0.41 & & & \\
\hline Baseline $\mathcal{E C T}$ & 0.91 & $0.83-0.99$ & 0.03 & 0.95 & $0.67-1.13$ & 0.06 \\
\hline Post-ablation ECT & 0.88 & $0.76-0.99$ & 0.05 & 0.84 & $0.66-1.07$ & 0.08 \\
\hline
\end{tabular}

Significant $\mathrm{p}$-values are shown in bold

$L A$, left atrium; $L A V$, left atrial volume; $R A$, right atrium; $E R$, reservoir strain; $E C T$, contractile strain

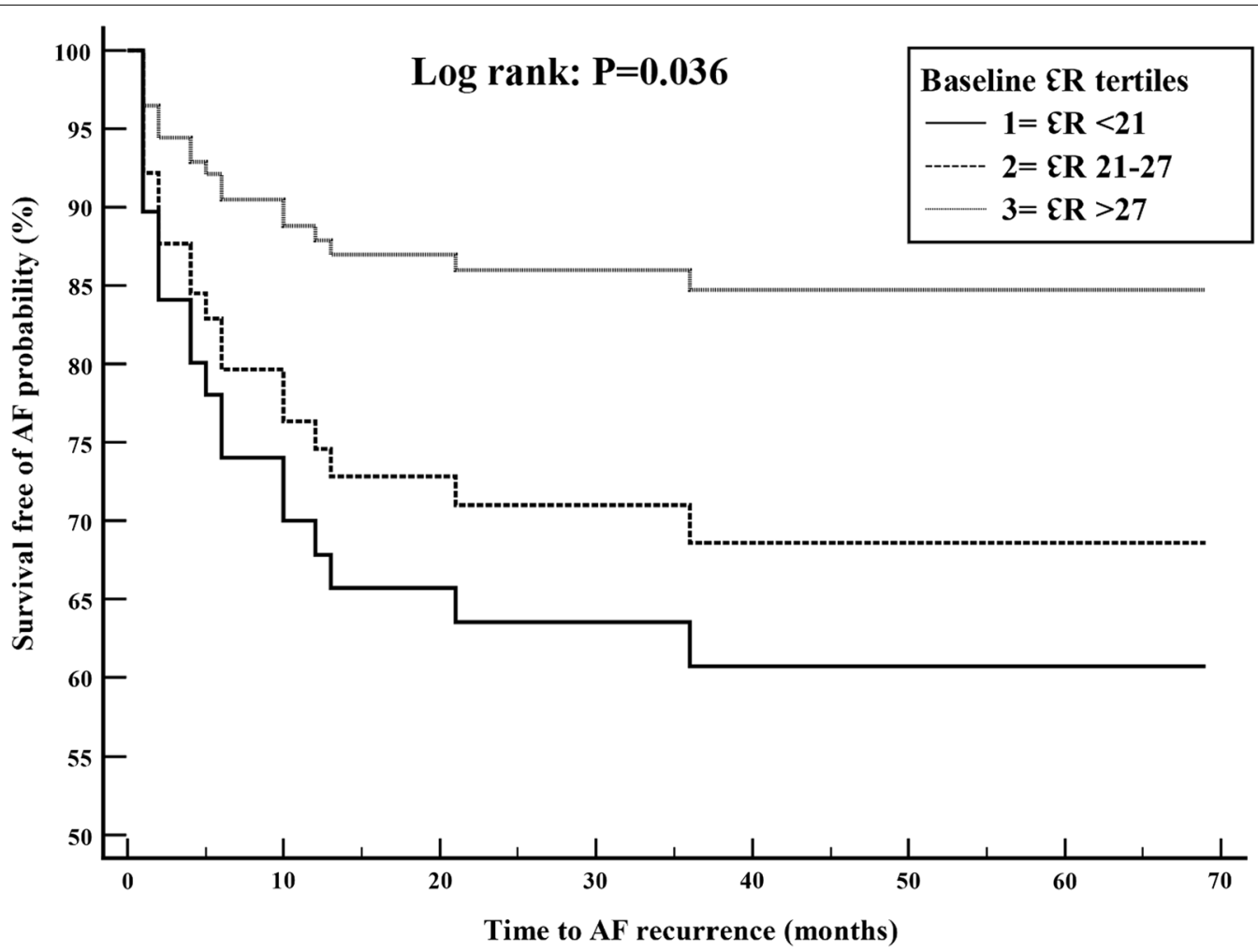

Fig. 2 Cox-proportional hazard model for survival free of atrial fibrillation by tertiles of baseline reservoir strain (ER), adjusted for age, gender, hypertension, and obstructive sleep apnea. Tertiles one, two, three corresponded to a baseline $\mathcal{E} R$ of $<21,21-27$ and $>27$, respectively 


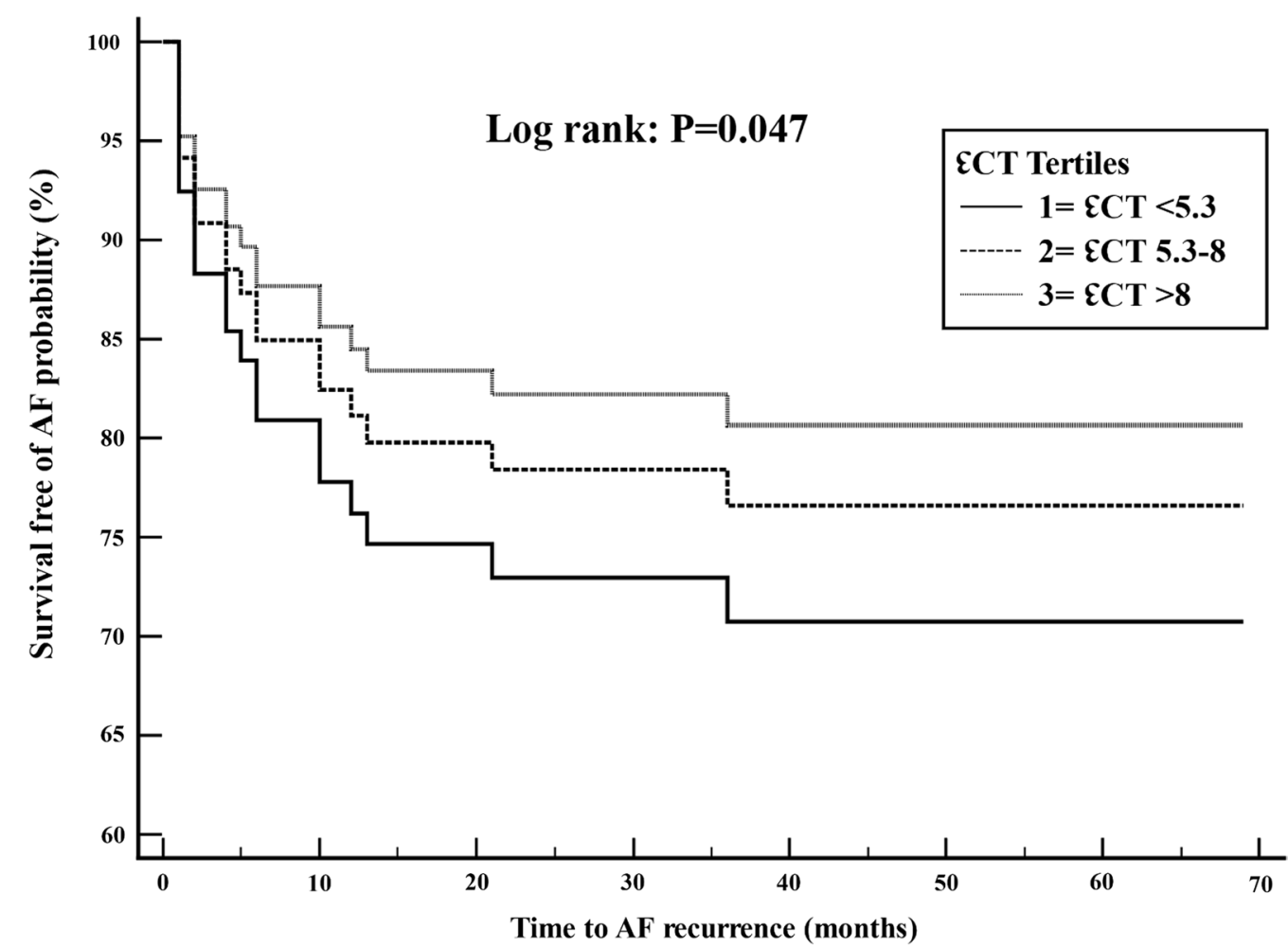

Fig. 3 Cox-proportional hazard model for survival free of atrial fibrillation by tertiles of post-ablation contractile strain (ECT), adjusted for age, gender, hypertension, and obstructive sleep apnea. Tertiles one, two, three corresponded to a post-ablation $\mathcal{E C T}$ of $<5.3,5.3-8$ and $>8$, respectively

risk of recurrence might need closer surveillance, more intense risk factor modification and/or modification of their antiarrhythmic regimen. Further validation studies are needed before CMR parameters can be included in the routine risk models in AF patients.

\begin{abstract}
Abbreviations
ECD: Conduit strain; $E C T$ : Contractile strain; $E R$ : Reservoir strain; 2DE: Two dimensional echocardiography; 3DE: Three dimensional echocardiography; AF: Atrial fibrillation; CMR: Cardiovascular magnetic resonance; ECG: Electrocardiography; LA: Left atrium; LAEF: Left atrial ejection fraction; LAV: Left atrial volume; LAVmax: Maximal left atrial volume; LAVmin: Minimum left atrial volume; LAVpreA: Pre atrial systole left atrial volume; LV: Left ventricle/left ventricular; LVEDVI: Left ventricular end-diastolic volume index; LVESVI: Left ventricular end-systolic volume index; pAF: Paroxysmal atrial fibrillation; PVI: Pulmonary vein isolation; RA: Right atrium/right atrial; RV: Right ventricular/ right ventricular; RVEDVI: Right ventricular end-diastolic volume index; RVESVI: Right ventricular end-systolic volume index.
\end{abstract}

\section{Supplementary Information}

The online version contains supplementary material available at https://doi. org/10.1186/s12968-021-00831-3.

Additional file 1: Multiple comparison graphs of the baseline and post-ablation reservoir (top) and contractile (bottom) strain data in both groups.

\section{Acknowledgements}

Not applicable.

\section{Authors' contributions}

MMB and MAS had full access to all of the data in the study and takes responsibility for the integrity of the data and the accuracy of the data analysis. Study concept and design: MAS, MMB. Acquisition of data: NM, MMB, AW, HR, NS. Analysis and interpretation of data: MMB, MAS. Draft of the manuscript: MMB, MAS. Critical revision of the manuscript for important intellectual content: MR, MK, TS, DW, MAS. Statistical analysis: MMB. Study supervision: MAS. All authors read and approved the final manuscript.

\section{Funding}

This study did not receive any funding.

Availability of data and materials

The datasets used and/or analysed during the current study are available from the corresponding author on reasonable request.

\section{Declarations}

Ethics approval and consent to participate

Ethics approval and informed consent was waived since this was a retrospective study.

\section{Consent for publication}

The manuscript is approved by all authors for publication.

\section{Competing interests}

The authors declare that they have no competing interests. 


\section{Author details}

${ }^{1}$ Division of Cardiovascular Medicine, Loyola University Medical Center, Stritch School of Medicine, Maywood, IL, USA. ${ }^{2}$ Department of Internal Medicine, Loyola University Medical Center, Maywood, IL, USA. ${ }^{3}$ Department of Internal Medicine, MacNeal Hospital, Berwyn, IL, USA.

Received: 17 March 2021 Accepted: 23 November 2021 Published online: 03 January 2022

\section{References}

1. Atrial fibrillation: current understandings and research imperatives. The National Heart, Lung, and Blood Institute Working Group on Atrial Fibrillation. J Am Coll Cardiol 1993;22:1830-4.

2. Leong DP, Eikelboom JW, Healey JS, Connolly SJ. Atrial fibrillation is associated with increased mortality: causation or association? Eur Heart J. 2013;34:1027-30

3. Corradi D, Callegari S, Benussi S, et al. Regional left atrial interstitial remodeling in patients with chronic atrial fibrillation undergoing mitralvalve surgery. Virchows Arch. 2004;445:498-505.

4. Sievers B, Kirchberg S, Addo M, Bakan A, Brandts B, Trappe HJ. Assessment of left atrial volumes in sinus rhythm and atrial fibrillation using the biplane area-length method and cardiovascular magnetic resonance imaging with TrueFISP. J Cardiovasc Magn Reson. 2004;6:855-63.

5. Kuppahally SS, Akoum N, Burgon NS, et al. Left atrial strain and strain rate in patients with paroxysmal and persistent atrial fibrillation: relationship to left atrial structural remodeling detected by delayed-enhancement MRI. Circ Cardiovasc Imag. 2010;3:231-9.

6. Margulescu AD, Nunez-Garcia M, Alarcon F, et al. Reproducibility and accuracy of late gadolinium enhancement cardiac magnetic resonance measurements for the detection of left atrial fibrosis in patients undergoing atrial fibrillation ablation procedures. Europace. 2019;21:724-31.

7. Nedios S, Koutalas E, Sommer P, et al. Asymmetrical left atrial remodelling in atrial fibrillation: relation with diastolic dysfunction and long-term ablation outcomes. Europace. 2017;19:1463-9.

8. Hundley WG, Meshack BM, Willett DL, et al. Comparison of quantitation of left ventricular volume, ejection fraction, and cardiac output in patients with atrial fibrillation by cine magnetic resonance imaging versus invasive measurements. Am J Cardiol. 1996;78:1119-23.

9. Kriatselis C, Unruh T, Kaufmann J, et al. Long-term left atrial remodeling after ablation of persistent atrial fibrillation: 7-year follow-up by cardiovascular magnetic resonance imaging. J Interv Card Electrophysiol. 2020:58:21-7.

10. Jahnke C, Fischer J, Gerds-Li JH, et al. Serial monitoring of reverse left-atrial remodeling after pulmonary vein isolation in patients with atrial fibrillation: a magnetic resonance imaging study. Int J Cardiol. 2011:153:42-6.

11. Dodson JA, Neilan TG, Shah RV, et al. Left atrial passive emptying function determined by cardiac magnetic resonance predicts atrial fibrillation recurrence after pulmonary vein isolation. Circ Cardiovasc Imag. 2014;7:586-92.

12. Mouselimis D, Tsarouchas AS, Pagourelias ED, et al. Left atrial strain, intervendor variability, and atrial fibrillation recurrence after catheter ablation: a systematic review and meta-analysis. Hellenic J Cardiol. 2020;61:154.

13. Tao S, Ciuffo LA, Lima JAC, Wu KC, Ashikaga H. Quantifying left atrial structure and function using single-plane tissue-tracking cardiac magnetic resonance. Magn Reson Imag. 2017:42:130-8.

14. Kowallick JT, Kutty S, Edelmann F, et al. Quantification of left atrial strain and strain rate using Cardiovascular Magnetic Resonance myocardial feature tracking: a feasibility study. J Cardiovasc Magn Reson. 2014;16:60

15. Truong VT, Palmer C, Wolking S, et al. Normal left atrial strain and strain rate using cardiac magnetic resonance feature tracking in healthy volunteers. Eur Heart J Cardiovasc Imag. 2020;21:446-53.

16. Pathan F, Zainal Abidin HA, Vo QH, Zhou H, D'Angelo T, Elen E, et al. Left atrial strain: a multi-modality, multi-vendor comparison study. Eur Heart J Cardiovasc Imag. 2021;22(1):102-10.

17. Badano LP, Kolias TJ, Muraru D, et al. Standardization of left atrial, right ventricular, and right atrial deformation imaging using two-dimensional speckle tracking echocardiography: a consensus document of the EACVI/
ASE/Industry Task Force to standardize deformation imaging. Eur Heart J Cardiovasc Imag. 2018;19:591-600.

18. Zareian M, Ciuffo L, Habibi M, et al. Left atrial structure and functional quantitation using cardiovascular magnetic resonance and multimodality tissue tracking: validation and reproducibility assessment. J Cardiovasc Magn Reson. 2015;17:52.

19. Ganesan AN, Shipp NJ, Brooks AG, et al. Long-term outcomes of catheter ablation of atrial fibrillation: a systematic review and meta-analysis. J Am Heart Assoc. 2013:2:e004549.

20. Andrade JG, Khairy P, Macle L, et al. Incidence and significance of early recurrences of atrial fibrillation after cryoballoon ablation: insights from the multicenter Sustained Treatment of Paroxysmal Atrial Fibrillation (STOP AF) Trial. Circ Arrhythm Electrophysiol. 2014;7:69-75.

21. Habibi M, Chahal H, Opdahl A, et al. Association of CMR-measured LA function with heart failure development: results from the MESA study. JACC Cardiovasc Imag. 2014;7:570-9.

22. Thomas L, Marwick TH, Popescu BA, Donal E, Badano LP. Left atrial structure and function, and left ventricular diastolic dysfunction: JACC state-of-the-art review. J Am Coll Cardiol. 2019;73:1961-77.

23. Tao S, Ashikaga $H$, Ciuffo LA, et al. Impaired left atrial function predicts inappropriate shocks in primary prevention implantable cardioverterdefibrillator candidates. J Cardiovasc Electrophysiol. 2017;28:796-805.

24. Zhuang J, Wang Y, Tang K, et al. Association between left atrial size and atrial fibrillation recurrence after single circumferential pulmonary vein isolation: a systamatic review and meta-analysis of observational studies. Europace. 2012;14:738-45.

25. Njoku A, Kannabhiran M, Arora R, et al. Left atrial volume predicts atrial fibrillation recurrence after radiofrequence ablation: a meta-analysis. EP Europace. 2018;20:33-42.

26. Habibi M, Samiei S, Ambale Venkatesh B, et al. Cardiac magnetic resonance-measured left atrial volume and function and incident atrial fibrillation: results from MESA (Multi-Ethnic Study of Atherosclerosis). Circ Cardiovasc Imag. 2016. https://doi.org/10.1161/CIRCIMAGING.115. 004299.

27. Ma XX, Zhang YL, Hu B, et al. The usefulness of global left atrial strain for predicting atrial fibrillation recurrence after catheter ablation in patients with persistent and paroxysmal atrial fibrillation. Arch Cardiovasc Dis. 2017:110:447-55

28. Cameli M, Mandoli GE, Loiacono F, Dini FL, Henein M, Mondillo S. Left atrial strain: a new parameter for assessment of left ventricular filling pressure. Heart Fail Rev. 2016;21:65-76.

29. Tops LF, Delgado V, Bertini M, et al. Left atrial strain predicts reverse remodeling after catheter ablation for atrial fibrillation. J Am Coll Cardiol. 2011;57:324-31.

30. Mirza M, Caracciolo G, Khan U, et al. Left atrial reservoir function predicts atrial fibrillation recurrence after catheter ablation: a two-dimensional speckle strain study. J Interv Card Electrophysiol. 2011;31:197-206.

31. Hammerstingl C, Schwekendiek M, Momcilovic D, et al. Left atrial deformation imaging with ultrasound based two-dimensional speckletracking predicts the rate of recurrence of paroxysmal and persistent atrial fibrillation after successful ablation procedures. J Cardiovasc Electrophysiol. 2012;23:247-55.

32. Cameli M, Lisi M, Righini FM, et al. Usefulness of atrial deformation analysis to predict left atrial fibrosis and endocardial thickness in patients undergoing mitral valve operations for severe mitral regurgitation secondary to mitral valve prolapse. Am J Cardiol. 2013;111:595-601.

33. Motoki H, Negishi K, Kusunose K, et al. Global left atrial strain in the prediction of sinus rhythm maintenance after catheter ablation for atrial fibrillation. J Am Soc Echocardiogr. 2014;27:1184-92.

\section{Publisher's Note}

Springer Nature remains neutral with regard to jurisdictional claims in published maps and institutional affiliations. 